Meta

Journal des tradlucteurs

Translators' Journal

\title{
Regards sur la traduction non littéraire de langue française
}

Henri Van Hoof

Volume 27, numéro 2, juin 1982

URI : https://id.erudit.org/iderudit/003079ar

DOI : https://doi.org/10.7202/003079ar

Aller au sommaire du numéro

Éditeur(s)

Les Presses de l'Université de Montréal

\section{ISSN}

0026-0452 (imprimé)

1492-1421 (numérique)

Découvrir la revue

Citer cet article

Van Hoof, H. (1982). Regards sur la traduction non littéraire de langue française. Meta, 27(2), 173-184. https://doi.org/10.7202/003079ar d'utilisation que vous pouvez consulter en ligne.

https://apropos.erudit.org/fr/usagers/politique-dutilisation/ 


\title{
Regards sur la traduction non littéraire de langue française
}

\author{
Henri Van Hoof
}

Dans un article consacré à la traduction médicale au cours des siècles ${ }^{1}$, nous avons pu montrer que la traduction scientifique avait des lettres de noblesse aussi anciennes que la traduction littéraire. Son importance avait été reconnue très tôt. Qu'il suffise de rappeler que, dès 146 av. J.-C., le sénat romain avait ordonné de traduire l'ouvrage consacré à l'agriculture par le Carthaginois Magon; que Nestorius (380-440), patriarche de Byzance, traduisit en syriaque les anciens textes médicaux grecs; que le calife abasside Al-Mamoun (786-834) organisa la traduction en arabe des œuvres scientifiques et philosophiques grecques; que l'archevêque Raymond de Tolède (1125-1151) créa un collège de traducteurs qui mirent en latin une grande partie du patrimoine arabe et grec portant sur les mathématiques, la physique, l'astronomie, l'alchimie, la médecine et la philosophie.

L'avènement des langues vulgaires en Occident n'a fait que renforcer cette nécessité de traduire aussi les ouvrages scientifiques. Nous nous limiterons ici au domaine français, où elle se manifesta dès le xve siècle par des traductions essentiellement médicales : la Chirurgia magna de l'Italien Lanfranco, traduite par le médecin lyonnais Guillaume Yvoire en 1479; la Cyrurgia de Guillaume de Saliceto, traduite par Nicolas Prévost en 1492; la Chirurgia magna de Guy de Chauliac, mise en français pour la première fois en 1497.

À partir du Xvie siècle, l'éventail des centres d'intérêt s'élargit et les traductions couvrent les champs d'activité les plus divers, des sciences naturelles à l'art, de la navigation à l'histoire, des sciences exactes à la psychologie. Pour chacune de ces disciplines, la production ira s'amplifiant au fil des ans, comme le montrera le panorama que nous en esquisserons jusqu'à l'aube de la période contemporaine.

\section{SCIENCES NATURELLES}

Cette rubrique ne reprend que les ouvrages consacrés aux aspects généraux de ce groupe de sciences et à certaines disciplines moins traduites; les disciplines qui ont bénéficié d'un développement plus important au cours des âges feront l'objet d'une rubrique séparée.

Avant même qu'il ne fût question de science, les livres sur la nature ont toujours tenté le lecteur et le traducteur. En 1564 déjà, Antoine Dupinet publie

1. META, vol. $26, \mathrm{n}^{\circ}$ 3, p. 215-222. 
l'Histoire naturelle de Pline traduite en françois avec un traité de poids et mesures antiques réduits à la façon des François. Trois ans plus tard, c'est Jacques Gohorry qui traduit les Occultes Merveilles et secrets de la Nature (1567) du médecin hollandais Levin Lesme. Pierre Amboise met en français l'Histoire naturelle (1631) de Francis Bacon. En 1803 paraît une version anonyme de l'Histoire naturelle et physiologique du biologiste italien Lazaro Spallanzani (1729-1799). En 1869, l'Essay of Classification du naturaliste suisse Louis Agassiz (1807-1873) est traduit sous le titre l'Espèce et les classifications. Puis se succèdent les traductions de trois ouvrages du naturaliste allemand Ernst Haeckel (1834-1919: Histoire de la création des êtres organisés d'après les lois naturelles (1874), Anthropogénie ou histoire de l'évolution humaine (1877) et les Preuves du transformisme (1879). La fin du siècle voit paraître l'ouvrage du physiologiste anglais Thomas Huxley (1825-1895), Evidence as to Man's Place in Nature, sous le titre de Place de l'homme dans la nature (1891).

En regardant d'un peu plus près le champ de la botanique, de l'agriculture et de la sylviculture, on note que dès 1533 Antoine Vérard publie une traduction de l'italien intitulée Livre des Prouffits champestres et ruraux. En 1720 paraît une version de l'Agriculture parfaite ou nouvelles découvertes du médecin allemand Georg Agricola (1672-1738). Buffon traduit la Statique des végétaux (1735), publiée en 1727 par le physicien et naturaliste anglais Stephen Hales (1677-1761). Du même Hales, Dupuy Demportes donne le Gentilhomme cultivateur (1765), tandis que Frenais, par ailleurs traducteur de Sterne, met en français les deux volumes du Guide du fermier (1770) de l'agronome anglais Arthur Young (1741-1820). En 1775, la Physiologica muscorum (1774) du botaniste allemand Noel Necker (1729-1793) paraît sous le titre de Physiologie des corps organisés. Au xIX siècle, on semble s'intéresser à des cultures bien précises si l'on en juge par des traductions comme le Traité de la culture des arbres fruitiers (1808) de l'horticulteur écossais William Forsyth (1737-1804) par Picket-Mallet, le Traité complet sur le sucre européen de betterave (1812) du chimiste allemand Franz Achard (17531821), l'Art de cultiver les mûriers (1826) de l'agronome italien Charles Verri (1743-1823), le Résumé des principaux traités chinois sur la culture des mûriers et l'éducation des vers à soie (1837) traduit par l'orientaliste Stanislas Julien, et les Principes de la science forestière (1841) du sylviculteur allemand Heinrich Cotta (1736-1844).

En zoologie, on peut citer la traduction d'un ouvrage de la naturaliste suisse Marie Merian (1647-1717), Der Raupen wunderbare Verwandlung, qui parait en 1730. Du même auteur, la Metamorphosis insectorum Surinamensium, publiée en 1705, est traduite en 1771 sous le titre Histoire générale des insectes de Surinam et de toute l'Europe. Dans le dernier quart du XIXe siècle, une équipe de savants, parmi lesquels Gerbe, Künckel, d'Herculaïs, Trémeau de Rochebrune, Sauvage, Constantin et Verneau, adaptent les treize volumes de la Vie illustrée des animaux, du naturaliste allemand Alfred Brehm (1829-1884), sous le titre les Merveilles de la nature.

Alors qu'en météorologie, on trouve déjà des traductions anonymes, l'une en 1556 des Dialogues sur certains points de la philosophie naturelle et choses météorologiques d'Antonio Luccioli, une autre en 1649 de l'Histoire des vents, où 
il est traité de leurs causes et de leurs effets de Francis Bacon, et une autre encore de la Loi des orages (1857) du physicien allemand Heinrich Dove (1803-1879) il faut évidemment attendre le début du XIXe siècle et la création de la paléontologie par Cuvier pour voir paraître aussi les premières traductions relatives à cette science nouvelle: en 1865, Demole traduit le Monde primitif de la Suisse du paléontologiste helvétique Oswald Heer (1809-1880) et, de 1883 à 1886, Charles Barrois donne sa version du Traité de paléontologie que le géologue allemand Karl von Zittel (1839-1904) avait publié quelques années plus tôt, en 1876.

\section{C.HIMIE}

Dans les premiers temps, c'est bien sûr d'alchimie qu'il est question, plus que de chimie. En 1548, François Habert traduit le Chrysopeion que l'alchimiste italien Giovanni Augurelli (1454-1537) avait publié en 1515. En 1612, c'est le Traité de la pierre philosophale d'un alchimiste juif arabe, Artéphius (xuI $\mathrm{s}$.), qui est mis en français par Pierre Arnauld.

A partir du xvirie siècle, c'est de chimie qu'il s'agit et, en 1788, Madame Lavoisier traduit l'Essai sur la phlogistique et sur la composition des acides du chimiste et minéralogiste irlandais Richard Kirwan (1735-1812). En 1829 paraît une traduction des Mémoires de physique, de chimie et de minéralogie du chimiste suédois Jean-Jacques Berzelius (1779-1848). De Berzelius encore, le médecin et savant Jean Hoefer traduit le Traité de chimie de 1846 à 1850 . Les Éléments de chimie expérimentale du Britannique $\mathrm{H}$. William paraissent en 1834 dans une version du chimiste parisien Henri Gaultier de Claubry (1792-1878).

\section{MINÉRALOGIE}

Il faut attendre le XVIII ${ }^{e}$ siècle pour voir apparaître les premières traductions dans cette discipline connexe de la chimie. En 1751, l'abbé Nicolas LengletDufresnoy, polygraphe érudit, diplomate, traduit l'Arte de los metales publié en 1640 par le minéralogiste espagnol Alvarez Barba, sous le titre de Métallurgie ou l'art de tirer et de purifier les métaux. En 1771 paraît une traduction de l'Essai d'une nouvelle minéralogie, publié en 1758 par le chimiste et minéralogiste suédois Axel Cronstedt (1722-1765). En 1784, J. Gibelin traduit les Éléments de minéralogie, de l'Irlandais Kirwan, déjà cité, ouvrage qui présente le premier classement des minéraux selon leur composition chimique. Le début du siècle suivant, c'est la Théorie de la terre (1815) du chimiste et géologue anglais James Hutton (1726-1797) qui voit le jour dans une version française.

\section{MATHÉMATIQUES}

En 1585 déjà paraît une traduction française du traité $D e$ Thiende, dans lequel Simon Stévin (1548-1620), le mathématicien du prince d'Orange, étudie les fractions décimales; publiée sous le titre de la Disme, elle constitue vraisemblablement la première traduction scientifique du néerlandais en français. Les Oeuvres mathématiques complètes de Stévin seront traduites par A. Girard en 1634.

En 1761, G.Fr. Castillon met en français l'Arithmétique universelle de Newton (1642-1727) et, quelques années plus tard, ce sont les Institutions analyti- 
ques de la mathématicienne italienne Maria Agnessi (1718-1799) qui paraissent en traduction.

Au XVIII siècle, les ouvrages traduits se multiplient, en provenance des horizons les plus variés. En 1811, ce sont les Principes de mathématiques publiés en 1782 par le Portugais Jose da Cunha (1744-1787). En 1836, Mallieux donne les Nouveaux fondements de la géométrie du Russe Nicolas Lobatcherski (17931856) et, quatre ans plus tard, c'est un autre ouvrage du même auteur, les $R e$ cherches géométriques sur la théorie des parallèles (1840), qui est traduit par J. Houël. Sur la fin du siècle, Dewulf met en français les Éléments de calcul graphique (1875) que l'Italien Luigi Cremona (1830-1903) avait publiés un an plus tôt. Un autre ouvrage du même auteur sort en 1885 , sous le titre de Figures réciproques dans la statique graphique, dans une version de Bossut. Une traduction des Éléments de calcul du mathématicien et astronome indien Aryabhata (ve s.) paraît en 1879, suivie en 1882 de celle du Traité élémentaire des quaternions publié en 1867 par le mathématicien anglais Peter Tait (1831-1901).

\section{ASTRONOMIE}

En astronomie aussi, on trouve déjà une traduction française au xvie siècle : il s'agit de la Cosmographie universelle (1575) de l'Allemand Sebastian Munster (1489-1552), due au très prolifique François de Belleforest. Puis, après une éclipse apparemment assez longue, trois traductions retiennent l'attention au $\mathrm{XIX}^{\mathbf{e}}$ siècle. La première est celle que V. Mauvais donne en 1845 du Mémoire sur la détermination des perturbations absolues dans les ellipses d'une excentricité et d'une inclinaison quelconques, de l'astronome allemand Peter Hansen (17951874). La deuxième est 1'Analyse spectrale des corps célestes de l'astronome anglais William Huggins (1824-1910), publiée en 1866 par le savant jésuite français Moigno, spécialiste de la traduction scientifique. La troisième, assez curieusement, est un retour à l'antiquité, avec la version du Traité $d u$ ciel d'Aristote, que Barthélémy-Saint-Hilaire donne en 1865.

\section{PHYSIQUE}

Dans le domaine de la physique, les traductions commencent tôt et suivent ensuite pas à pas le développement de ses diverses branches. En 1556 déjà, Richard Le Blanc, par ailleurs interprète de Virgile, donne les Livres de Hierosme Cardan intitulez de la Subtilité et Subtiles inventions, dans lequel le savant et philosophe Jérome Cardan (1501-1576) exposait sa théorie physique de la nature. En 1664 parait en traduction De la mesure des eaux courantes, publiée en 1638 par le mathématicien et physicien italien Benoit Castelli (1577-1644). En 1673, Deux traités: De la nature des éléments; De la quintessence du physicien hollandais Cornelius van Drebbel (1572-1634), parus en 1608, sont à leur tour mis en français et cette version française servira de base à une traduction latine intitulée Tractatus duo: De natura elementarium; De quinta essentia (1621).

Au xviri ${ }^{e}$ siècle, le rythme des traductions s'accélère. Une version anonyme de l'Optique de Newton paraît en 1720, bien avant la traduction signée par Marat (1787). En 1740, Lemonnier met en français les Lectures sur l'hydrostatique et la pneumatique, du mathématicien et astronome anglais Roger Cotes (1682-1716), 
sous le titre de Leçons de physique expérimentale. En 1757 paraît une traduction des Éléments de physique de John Locke (1632-1704), philosophe anglais que le souci de la réalité avait amené à s'occuper de physique, de chimie et de médecine. Raoul traduit les Réflexions sur la distribution de la chaleur à la surface de la terre (1762) du physicien allemand Franz Hoch, dit Aepinus (1724-1802). Un $R e$ cueil des auvres physiques de Richard Mead (1673-1754), médecin du roi George III d'Angleterre, paraît dans une traduction anonyme en 1764. Le physicien Mathurin Brisson donne en 1771 une traduction de l'Histoire et l'état présent de l'électricité, publiée en 1765 par le chimiste et philosophe anglais Joseph Priestley (1733-1804), dont l'abbé Jean-François Rozier, agronome et botaniste, traduit encore les Observations sur différentes espèces d'air parues en 1772. Du même auteur encore, les quatre volumes des Expériences et observations sur la physique sont mis en français de 1782 à 1787 par J. Gibelin, déjà cité. Et, de Priestley encore, P.A. Adet traduit les Réflexions sur la doctrine du phlogistique et la décomposition de l'eau (1798).

Et si la série se poursuit au $\mathrm{XIX}^{\mathrm{e}}$ siècle avec des traductions qui trahissent davantage les progrès mécaniques et les préoccupations de l'époque, telle celle du Traité de l'économie des machines et des manufactures du mathématicien anglais Charles Babbage (1792-1871) par Biot, celle du Traité des distributions par tiroirs dans les machines à vapeur fixes et les locomotives (1869) de l'ingénieur allemand Gustav Zeuner (1828-1907) par un anonyme, ou celle encore, par l'abbé Moigno déjà cité, de l'ouvrage $O$ On the correlation of physical forces (1856) publié dix ans plus tôt par le physicien anglais William Grove (1811-1896), on trouve même un Jules Barthélémy-Saint-Hilaire, homme politique et philosophe, pour s'atteler encore à la Physique (1862) et à la Météorologie (1863) d'Aristote.

\section{ARCHITECTURE, ARTS}

Les traducteurs français se sont intéressés très tôt aux ouvrages d'architecture. De J. Martin, on possède une traduction des Six livres d'architecture (1545) de l'Italien Serbio, une autre des Huit livres d'architecture (1547) de l'architecte romain Marcus Vitruve (88-26 av. J.-C.), une autre encore de l'Architecture (1553) de l'humaniste italien Leo-Battista Alberti (1404-1472). En 1557 paraissent Les quatre livres d'Albert Dürer, peintre et géomètre très excellent, dans une version de Louis Meigret, qui fut aussi un traducteur infatigable des Anciens.

Au siècle suivant, on relève une traduction des Quatre livres d'architecture (1650) de l'italien Andrea Palladio (1518-1580) sous la plume de F. de Chambray. Puis, c'est une version plus complète de Vitruve qui paraît sous le titre les Dix livres d'architecture (1673) par les soins de Claude Perrault, médecin et architecte à qui l'on doit la colonnade du Louvre. Et, en 1691, Daviler donne le Traité d'es cinq ordres d'architecture de l'italien Giacomo Barozzi, dit Vignole (15071573).

$\mathrm{Au}$ XVIII siècle, ce sont surtout les arts plastiques qui mobilisent l'attention. Bergier traduit les Recherches sur les beautés de la peinture (1765) de l'Anglais D. Webb. L'année suivante, Sellius met en français l'Histoire de l'art chez les Anciens (1766) de l'archéologue allemand Johann Winckelmann (1717-1768), dont 
Jansen donne encore les Remarques sur l'architecture des Anciens (1783). Gérard de Lairesse traduit les deux volumes du Grand livre des peintres, ou l'Art de la peinture considéré dans toutes ses parties, et, en 1791 paraît le Traité de la peinture de Léonard de Vinci (1452-1519), dans une version du critique d'art Pierre Gault de Saint-Germain.

L'intérêt pour les ouvrages d'art se manifeste encore au siècle suivant, avec une traduction de Madame A. Dieudé de l'Histoire de la peinture en Italie (1824), publiée en 1789 par le jésuite et archéologue italien Louis Lanzi (1732-1810), et deux autres de Claudius Popelin consacrées au Della statua et au De pictura (1868) de l'humaniste Alberti déjà cité.

\section{ART MILITAIRE}

Les arts de la guerre n'ont pas échappé à la curiosité des traducteurs. En 1546 déjà, Jean Charrier donne l'Art de la guerre de Machiavel. Dix ans plus tard paraît une traduction de la Pirotecnia, premier livre sur la fonte des canons et la fabrication de la poudre, publié en 1540 par le savant italien Vanuccio Biringuccio (fin $\mathrm{XV}^{\mathrm{e}}-1^{\text {re }}$ moitié $\mathrm{XVr} \mathrm{r}^{\mathrm{s}} \mathrm{s}$.).

Deux siècles plus tard, c'est Louis Dupuy qui traduit les Nouveaux principes d'artillerie (1771) du mathématicien anglais Benjamin Robins (1707-1751), ouvrage qui connaît une nouvelle version en 1783 sous la plume de Lombard. Et, en 1775, Turgot publie sa traduction de l'allemand des Commentaires sur les principes d'artillerie de Robins, du mathématicien suisse Leonard Euler (1707-1783).

\section{NAVIGATION}

Dès 1584 paraît la traduction française d'un Art de la navigation en langue espagnole. En 1722, c'est une version anonyme de l'Histoire de la navigation de Locke; en 1751, une autre version anonyme de l'Histoire navale de l'Angleterre de Th. Lediard. Et, en 1775, Turgot traduit le Traité de la construction et de la manœuvre des vaisseaux du Suisse Euler déjà cité.

\section{GÉOGRAPHIE}

De la navigation à la géographie, il n'y a qu'un pas, car du Xve au XIX siècle, tous les voyages se font évidemment par la voie maritime, et les ouvrages de géographie qui paraissent sont essentiellement des relations de voyages. Les innombrables traductions qui en sont faites retracent en quelque sorte la découverte du monde. Elles commencent donc très tôt, avec Mathurin du Redouer qui publie en 1516 le Nouveau Monde et les navigations faites en tous temps par Emery Vespuce, du navigateur florentin Amerigo Vespucci (1452-1529). En 1556, Louis Temporal traduit le Voyage de Loys Bartheme, Bolognais, du voyageur Luigi Barthema, dit Vartomanus (1480-?).

Dès le XVIIe siècle, l'horizon s'élargit avec Van Baerl, qui met en français la Description des Indes occidentales (1622) de l'historien espagnol Antonio de Herrera y Tordesillas (1559-1625) et les Voyages aventureux de Fernand Mendez Pinto, fidèlement traduits du portugais en français par le Sieur Bernard Figuier, gentilhomme portugais. Dediez à Monseigneur le Cardinal de Richelieu (1645). Peu de temps après, Wicquefort fait connaître au public français les Relations de 
voyage en Moscovie, Tartarie et Perse (1656) de l'allemand Oelschlager et Perrot d'Alblancourt, le père des «belles infidèles», traduit la Description de l'Afrique (1667) de l'historien espagnol Luis del Marmol y Caraval (1520-?).

Dès le XviII siècle, c'est l'avalanche. En 1711 paraît une traduction des Voyages aux terres magellaniques du navigateur anglais Ambrose Cowley, qui accompagna le capitaine John Cook. Six ans plus tard, c'est l'État présent de la grande Russie ou Moscovie (1717) du médecin et voyageur anglais John Moore (1729-1802), dans une version de Hugony, suivi en 1718 d'une traduction anonyme du Voyage de Corneille Le Brun par la Moscovie, en Perse et aux Indes orientales, du Hollandais Cornelius Debruyn, dont l'abbé Barrier donne une nouvelle version dès 1725 . Un anonyme encore publie en 1722 le Voyage aux Indes occid'entales de l'espagnol Fr. Correal.

En 1735 paraît une traduction qui n'est pas une relation de voyage, mais un ouvrage véritablement géographique; c'est la Géographie physique ou Essai sur l'histoire naturelle de la terre de l'Anglais John Woodward, due au $\mathrm{Dr}$ Noguès. Mais dès 1743, la ronde des explorations reprend avec une traduction anonyme des Voyages dans plusieurs provinces de Barbarie et du Levant du voyageur anglais Thomas Shaw (1692-1751). L'académicien Jean-Baptiste Suard traduit indifféremment le Voyage autour du monde (1767) de Lord Byron et le Second voyage (1778) du navigateur anglais James Cook (1728-1779). En 1772, c'est encore une traduction anonyme des Voyages en Orient (Égypte, Syrie Grèce, etc.) de l'anglais J. Pococke, suivie d'une autre anonyme de la Description des terres magellaniques et des pays adjacents (1778), publiée quatre ans auparavant par le voyageur anglais Thomas Falkner (?-1780). En 1786, P.H. Mallet donne le Voyage en Pologne, Russie, Suède et Danemark du biographe anglais William Coxe (1747-1828), précepteur de plusieurs seigneurs whig qu'il accompagna dans leurs voyages en Europe. Le Voyage en Abyssinie, publié en 1790 par le voyageur écossais James Bruce (1730-1794), est mis en français l'année suivante par J. Castéra, qui, en collaboration avec Benoît, donne encore le Voyage à l'intérieur de l'Afrique (1799) d'un autre explorateur écossais, Mungo Park (1771-1806), mort par noyade au cours de sa descente du Niger. L'officier et orientaliste Louis Langlès traduit de 1795 à 1798 le Voyage d'Égypte et de Nubie du voyageur danois Frédéric Norden (1708-1742), ainsi que deux ouvrages du botaniste suédois Charles Thunberg (1743-1828), à savoir les Voyages en Europe, en Afrique et en Asie pendant les années 1770-1779 et les Voyages de C.P. Thunberg au Japon par le Cap de Bonne Espérance, les Isles de la Sonde, etc. (1796).

Le XVIII ${ }^{e}$ siècle s'ouvre sur plusieurs traductions anonymes: celle du Voyage en Abyssinie (1816) publiée en 1814 par l'explorateur anglais Henri Salt (11785-1827), celle du Second voyage à la recherche d'un passage nord-ouest (1835) de l'explorateur anglais John Ross (1777-1856). En 1838, A. de Montry et E. Fraissinet traduisent l'ouvrage du médecin allemand Philipp von Siebold, Voyage au Japon exécuté pendant les années 1823 à 1830 ou description physique, géographique et historique de l'empire japonais. Deux ouvrages de l'explorateur David Livingstone (1813-1873) paraissent en traduction anonyme : Voyages et recherches d'un missionnaire dans l'Afrique méridionale (1859) et Relation 
de l'exploration du Zambèze et de ses affluents (1866). Ce sont encore des anonymes qui traduisent The Great Frozen Sea (1880), publié en 1878 par l'explorateur anglais Albert Markham (1841-1918), et, Tombouctou, voyage à travers le Maroc, le Sahara et le Soudan (1886), publié en 1884 par le géologue et voyageur allemand Heinrich Lenz (1848-1925). En 1883-84, le géographe et voyageur Charles Rabot donne le Voyage de la «Vega» du naturaliste et explorateur suédois Nils Nordenskjoeld (1832-1901) et, en 1897, le Spitzberg traversé pour la première fois, du critique d'art et alpiniste anglais William Conway (1856-1937). Et le siècle se clôture par une traduction anonyme de plus, celle de The Forbidden Land (1899), publié un an plus tôt par l'explorateur anglais Henry Savage Landor (1865-1924), qui parcourut le Tibet, le Népal, la Corée, etc.

\section{HISTOIRE}

En matière de traduction, l'histoire suit la géographie dans la foulée, puisque dès le milieu du Xvie siècle, on trouve tout un éventail d'ouvrages, essentiellement espagnols, portugais ou italiens, qui sont ainsi mis à la portée du lecteur français : les Commentaires de la guerre de Charles-Quint contre les Protestants d'Allemagne (1551) du capitaine et historien Luis d'Avila y Zuniga, par l'académicien Gilles Bouleau; l'Histoire des Indes de Portugal (1553) de l'historien portugais Fernando Lopez de Castanheda, par Nicolas de Grouchy; l'Histoire des Indes (1571) de l'Italien Maffei, par Edmond Auger; le De Maria Scotorum Regis (1572) de l'humaniste et historien écossais George Buchanan (1506-1582), par Camuz; l'Histoire florentine (1577) de Machiavel par Yves de Brinon; l'Histoire nouvelle du monde (1579) de l'Italien Girolamo Benzoni, par Urbain Chauveton; les Tyrannies et cruautés des Espagnols perpétrées aux Indes occidentales (1579) du prélat espagnol, protecteur des Indiens, Barthélémy de Las Casas (1474-1566), par Jacques de Miggrodes.

$\mathrm{Au} \mathrm{XVII}$ siècle encore, ce sont les auteurs espagnols et italiens qui restent les plus traduits. La traduction que La Tour Hotman donne en 1627 de l'Histoire du règne de Henri VII, roi d'Angleterre, de messire Fr. Bacon fait quelque peu figure d'exception. L'Histoire du Concile de Trente (1621) de l'Italien Paolo Scarpi est mise en français par J. Diodati. Jean Baudoin, traducteur infatigable des Anciens et des classiques italiens, donne successivement l'Histoire des Incas (1633), de l'historien péruvien Garcilosa de la Vega (1535-1568), l'Histoire des guerres civiles de France (1642) publiée en 1630 par l'historien italien Enrico Davila (1576-1631), et les Guerres civiles des Espagnols dans les Indes (1650) de Garcilosa de la Vega encore. En 1659 paraît sous la plume de La Coste une traduction des Conquêtes des Espagnols aux Indes, de 1'Espagnol Antonio de Herrera, premier historiographe des Indes. En 1670, c'est Briot qui traduit le Tableau de l'état présent de l'Empire ottoman, publié deux ans plus tôt par le diplomate et historien anglais Paul Rycaut (1628-1700), dont il donnera encore l'Histoire de l'Empire ottoman, en six volumes, au début du siècle suivant (1709). En 1678 paraît une version anonyme de l'Histoire de la conquête de la Chine par les Tartares, de l'ecclésiastique espagnol Juan de Palafox de Mendoza (1600-1659). Rouxel traduit l'Introduction à l'histoire universelle (1685) du juriste et historien allemand Samuel von Pufendorf (1632-1694), dont toute l'œuvre est encore écrite 
en latin. Et le siècle se clôture sur la traduction d'un autre auteur espagnol, celle de l'Histoire de la conquête du Mexique ou de la Nouvelle Espagne (1691) du poète lyrique et historien Antonio de Solis (1610-1686) par un anonyme, et la traduction d'un autre ouvrage italien - mais écrit en latin - celle de l'Histoire de la Chine (1692) du missionnaire jésuite Martin Martini (1614-1661).

Le XVIII ${ }^{e}$ siècle voit les traducteurs se tourner davantage vers les auteurs allemands et anglais. Ainsi, de 1728 à 1742, D. de Laroque et l'abbé Guyon traduisent les seize volumes de l'Histoire romaine de l'historien anglais Laurence Echard (1670-1730). Dans l'entre-temps, Schurchzer donne l'Histoire naturelle, civile et ecclésiastique de l'Empire du Japon (1729) de l'Allemand Kempfer. L'abbé Antoine Prévost (1697-1763), par ailleurs traducteur de Steele et de Richardson, met en français l'Histoire de Cicéron (1743), publiée en 1741 par le théologien C. Middleton, et l'Histoire de la maison de Stuart jusqu'au détrônement de Jacques II (1760), du philosophe et historien David Hume (1711-1776). D'autres ouvrages de Hume, comme l'Histoire de la maison de Tudor (1763) et 1'Histoire de la maison de Plantagenet sur le trône d'Angleterre (1765) sont traduits par une certaine Madame Belot. L'Histoire d'Angleterre (1759), du romancier écossais George Smollett (1721-1771), paraît dans la version d'un certain Targe. L'académicien Suard, que nous connaissons déjà comme traducteur d'ouvrages géographiques, se penche aussi sur l'histoire et donne l'Histoire de Charles-Quint (1771) et l'Histoire de l'Amérique (1778), publiées respectivement en 1769 et 1777 par un autre Écossais, William Robertson (1721-1793). A la fin du siècle, A.S. d'Arnay publie une traduction de l'Histoire de la Guerre de Trente Ans (1794) du grand Friedrich Schiller. On le voit, les cuvres anglaises et allemandes dominent, mais on aurait pu signaler en passant, et surtout au début du siècle, quelques versions anonymes d'anciennes langues de prédilection, telles l'Histoire de la découverte et de la conquête du Pérou (1716) de l'Espagnol Fernando de Zarate; le Voyage historique d'Abyssinie (1728), traduit du portugais, du missionnaire portugais Jérome Lobo (1595-1678); et l'Histoire des guerres d'Italie (1738) du diplomate et historien florentin Francesco Giucciardini (1483-1540).

Cette même orientation des traducteurs se maintient au XIX ${ }^{e}$ siècle et, mise à part la traduction de l'Histoire universelle de l'Italien Cesare Cantu (1804-1895) que E. Aroux publie en dix-neuf volumes de 1848 à 1849 , on y relève essentiellement des ouvrages anglais et allemands. Campesson donne en 1820 une nouvelle œuvre de Robertson, son Histoire d'Écosse. L'année d'après, Benoist traduit l'Histoire de la Perse (1821) du général et diplomate anglais John Malcolm (1769-1833). L'Histoire romaine du diplomate allemand Berthold Niebuhr paraît en 7 volumes (1863-72) dans une version de l'érudit Marie-Philippe de Golbéry (1786-1854). L'Histoire d'Angleterre (1852), publiée en 1848 par le critique et historien Thomas Babington, lord Macaulay (1800-1859), est traduite par J. de Peyronnet. De 1869 à 1872, Alexandre donne en 8 volumes une traduction de l'Histoire romaine du plus illustre représentant des sciences historique et philosophique allemandes de la seconde moitié du XIXe siècle, l'érudit Theodor Mommsen (1817-1903). Enfin, Elias Regnault collabore avec le littérateur Odysse Barot (1830-1907) et avec l'homme politique Jules Roche (1841-1923) pour tra- 
duire les trois volumes de l'Histoire de la Révolution française (1865-66), que Thomas Carlyle (1795-1881) avait publiée en 1837.

PHILOSOPHIE, PSYCHOLOGIE, SOCIOLOGIE

La philosophie a toujours été une fidèle compagne du traducteur français. Mais c'était une compagne qui, jusqu'au Xvie siècle encore, parlait essentiellement le grec et, surtout, le latin qui était demeuré la langue des sciences. Qu'il suffise de rappeler qu'Étienne Dolet terminait une traduction des Oeuvres complètes de Platon, lorsqu'il fut condamné au bûcher en 1546. Et s'il paraissait quelques traductions de philosophes contemporains, des Italiens Ficin ou Pic de la Mirandole, de l'Allemand Melanchton ou d'autres, toutes procédaient cependant du latin, langue d'écriture choisie par ces auteurs.

Il faut donc attendre la fin du XVI ${ }^{e}$ siècle pour trouver les premières traductions d'œuvres philosophiques conçues en langue vulgaire, telle celle de l'Examen des esprits (1580) de l'Espagnol J. Huarte ou celle des Dialogues philosophiques de l'Italien Cinthio. Mais c'est au XVII et surtout au XVIII ${ }^{e}$ siècles que se produit le véritable essor, et dans une tout autre direction, car si Vion d'Alibray s'intéresse encore au monde latin en donnant une version nouvelle de l'Examen des esprits (1645), c'est à présent le monde anglo-saxon qui semble fasciner le traducteur. Après un Traité de la raison humaine (1682) de Clifford, traduit par Poppel, on note successivement l'Essai sur l'entendement humain (1700) de John Locke (1632-1704) par Pierre Costa, les Recherches philosophiques sur la liberté de l'homme (1720) du disciple de Locke Anthony Collins (1676-1729) par de Pons, les Principes de la philosophie morale (1744) de Shaftesbury (1671-1713) par Paillet, les Eléments de philosophie newtonienne (1755) de Pemberton par Elie de Joncourt, les Oeuvres philosophiques (1759, 5 vol.) de David Hume (1711-1776) par Mérian et Robinet, la Métaphysique de l'âme ou théorie des sentiments moraux (1764) d'Adam Smith (1723-1790) par Marc-Antoine Eidous, qui traduit aussi le Système de philosophie (1770) et le Traité des passions (1775) de l'Irlandais Francis Hutcheson (1694-1747) une nouvelle version de la Théorie des sentiments moraux (1790) de Smith par Roucher.

$\mathrm{Au} \mathrm{XIX}^{\mathrm{e}}$ siècle, le traducteur découvre les philosophes allemands. En 1833, Poret met en français un manuel intitulé Lehrbuch für den ersten Unterricht in der Philosophie, publié en 1823 par August Matthliae (1769-1835). La Philosophie de la vie de Friedrich von Schlegel (1772-1829) paraît en 1837 dans une version de Guénot. Pratiquement toute l'cuvre d'Emmanuel Kant (1724-1804) est traduite par deux hommes : le littérateur et philosophe Joseph Tissot en donne les Principes métaphysiques de la morale (1830), les Principes métaphysiques du droit (1839), la Religion dans les limites de la raison (1841), les Prolégomènes à toute métaphysique future (1865); de son côté, l'homme politique et philosophe Jules Barni publie la Critique de la raison pure (1835), les Éléments métaphysiques de la doctrine de la vertu (1843), la Critique du jugement (1846) et la Critique de la raison pratique (1848). Friedrich Hegel (1770-1831) trouve son interprète principal en la personne du philosophe italien Auguste Véra, professeur en France, qui traduit sa Logique (1859), sa Philosophie de la Nature (1863-65), sa Philosophie de l'esprit (1867-68) et sa Philosophie de la religion (1876-78). Les idées de Karl Marx 
(1818-1883) se répandent par des traductions comme Misère de la philosophie (1843) et le Capital (1873), par J. Roy. De Arthur Schopenhauer (1788-1860) paraissent l'Essai sur le libre arbitre (1876) par S. Reinack et aussi De la quadruple racine du principe de la raison suffisante (1881) et Le Monde comme volonté et comme représentation (1886), traduits par J.A. Cantacuzène. Une version anonyme de la Philosophie de l'inconscient du moniste Karl von Hartmann (18421906) voit le jour en 1877 et, la même année, Pommerol traduit l'Histoire du matérialisme, l'ouvrage capital de Friedrich Lange (1828-1875). Friedrich Nietzsche (1844-1900) et sa théorie du surhomme pénètrent en France par les traductions qu'Henri Albert donne de ses œuvres maîtresses : Ainsi parla Zarathoustra (1898), la Généalogie de la morale (1900), etc.

Cette avalanche de traductions de l'allemand n'a cependant pas éclipsé totalement les philosophes anglo-saxons. Même ceux d'Outre-Atlantique sont abordés, en la personne de Ralph Waldo Emerson (1803-1882), dont une première traduction, Essais de philosophie américaine (6 vol.), voit le jour de 1851 à 1854 par les soins du critique Émile Montégut. De John Stuart-Mill (1806-1871), P. L. Le Monnier donne l'Utilitarisme (1883). De Herbert Spencer (1820-1903) paraît l'Introduction à la science sociale (1874), tandis que les philosophes Théodule Ribot et Alfred Espinas mettent en français ses Principes de psychologie (1874-75) et que ses Principes de sociologie sont traduits de 1878 à 1887 par Émile Cazelles, médecin et philosophe, qui contribua puissamment à répandre les idées évolutionnistes en révélant les œuvres capitales de l'école anglaise.

Une exploration plus spécifique du domaine de la psychologie, constituée en science positive au cours du XIX $^{\mathrm{e}}$ siècle seulement, révèle essentiellement des traductions de l'allemand et de l'anglais. Penjon traduit les Principes de psychologie physiologique (1881), publiés en 1852 par l'Allemand Hermann Lotze (1817-1881). Deux livres du psychologue anglais James Sully (1842-1923), Pessimism, a history and a criticism (1877) et On Illusions, a psychological study (1881), paraissent sous les titres respectifs le Pessimisme, histoire et critique (1882) et les Illusions des sens et de l'esprit (1883). Rouvier donne les Principes de psychologie physiologique (1885) que l'Allemand Wilhelm Wundt (1832-1920) a publiés en 1874. Enfin, en 1897 et 1898, ce sont deux ouvrages du psychologue et sociologue américain James Baldwin (1861-1934), Mental Development in Child and Race et Social and Ethical Interpretation in Mental Development, qui sont mis à la portée du lecteur français.

En sociologie, également, ce sont les auteurs allemands et anglo-saxons qui priment. D'entrée de siècle, Dormant traduit les Considérations sur les rapports qui lient les hommes en société (1800) de l'Anglais J. Brown. Les Principes de la science sociale, publiés en 1858-60 par l'économiste américain Henry Carey (1793-1879), paraissent peu de temps après en français. Les Principles of Sociology du sociologue américain Franklin Giddings (1855-1931) sont traduits en 1896. Au tournant du siècle suivant, c'est l'État socialiste (1904) du juriste allemand Anton Menger (1841-1906), rallié aux doctrines de Marx et Engels.

Clôturons ici ce panorama d'une activité traduisante qui peut surprendre par sa diversité et son ampleur. Il nous montre, en tout cas, que le traducteur a toujours été présent à chaque progrès des sciences et des techniques, qu'il a suivi 
l'actualité technologique et sociologique du monde de plus en plus près, en un mot, qu'il fait partie intégrante du monde et qu'il n'en est pas que le spectateur. Et, à notre époque plus qu'à toute autre, le traducteur participe à la construction et à l'évolution du monde, la traduction pénètre toutes les activités. Comme a pu le dire le regretté Edmond Cary : «Dans le monde moderne, le savant, le technicien, l'homme d'État ne se réalisent qu'à travers dix, vingt traducteurs... Avec le $\mathrm{xx}^{\mathrm{e}}$ siècle, c'est au seuil d'un âge nouveau que nous arrivons, l'âge de la traduction par excellence ${ }^{2}$. 